\title{
Thermal Rearrangements in the Tetra-arylcyclopropene Series
}

\author{
By Merle A. Battiste*, Brian Halton, and Robert H. Grubbs \\ (Department of Chemistry, University of Florida, Gainesville, Florida 32601)
}

THE literature provides many examples of thermal rearrangements of small-ring compounds to yield systems involving less bond-angle strain. ${ }^{1}$ In the arylcyclopropene series these involve, in many cases, fairly complex pathways, and only formalized mechanisms have been suggested. ${ }^{\mathbf{1 , 2}}$ Tetra-arylcyclopropenes have the advantage of no labile hydrogen atom, exhibit moderate thermal stability, and would be expected to yield only primary products from ring cleavage. We now report that 3-aryl-1,2,3-triphenylcyclopropenes (I) readily available by action of the appropriate aryl Grignard reagent on triphenylcyclopropenyl bromide, rearrange, either in a melt above $200^{\circ}$ or in solution at somewhat lower temperatures, to 1,2,3-triarylindenes. The rearrangement also proceeds under acidic conditions at considerably lower temperature. $^{3}$

Molten tetraphenylcyclopropene ( $\mathrm{Ia})^{4}$ rearranged at $235-240^{\circ}$ in almost quantitative yield to $1,2,3-$ triphenylindene (IIa) The rearrangement exhibited first-order kinetics in diphenyl ether $(88 \%)$ quinoline $(12 \%)$ (See Table), but was sensitive to traces of acid and oxygen. $\uparrow$

$\dagger$ Analysis was by n.m.r. integration, at $120^{\circ}$, of the increasing 1 -indenyl proton $(\tau 4.93)$ against the aliphatic protons of fluorene $(\tau 6 \cdot 28)$ used as an internal standard. The average of 12 integrations was taken for each point. Erratic kinetic behaviour was observed for tubes sealed at pressures greater than $10^{-4} \mathrm{~mm}$. $\mathbf{H g}$, and with less than $12 \%$ quinoline in the solvent mixture. Since (IIa), as well as most triarylindenes, fluoresces strongly in the visible the rearrangement can also be conveniently followed by fluorimetry. 
Triphenylindene formation from (Ia) under thermal conditions can be formally written in three ways: (i) a homolytic cleavage of a ring carboncarbon single bond giving rise to a diradical intermediate; (ii) a heterolytic cleavage yielding ionic intermediates; and (iii) a concerted process. The

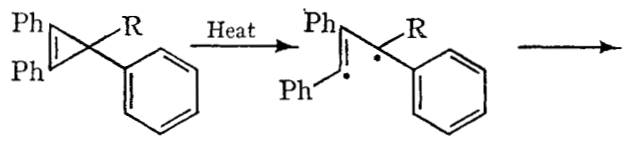

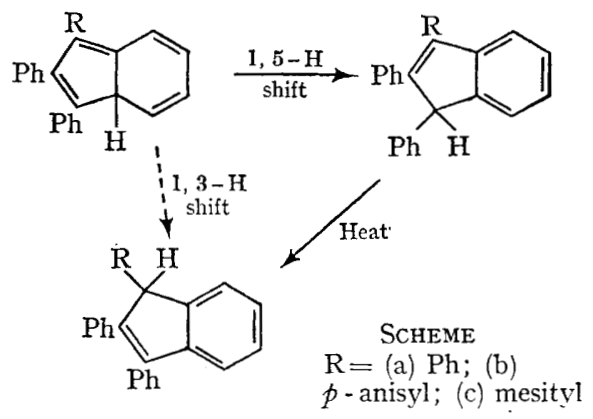

diradical route is shown in the Scheme. Aromatization of the intermediate isoindene (III) affords indene (II) by a thermally favoured 1,5-hydrogen shift, ${ }^{6}$ but (IV) may also arise by a series of 1,5 hydrogen shifts, before or after aromatization or directly by a less favoured $1,3(1,7)$-hydrogen shift. A similar set of indene products would result from ring opening followed by ring closure on to the 3aryl substituent. Preliminary investigations with 3-p-anisyl-1,2,3-triphenylcyclopropene (Ib), $\ddagger$ support this product scheme. Two major indene components, contaminated with isomeric impurities, are obtained from the thermal rearrangement of (Ib) at $200^{\circ}$.

With the ortho-positions of the aryl group of (I) formally blocked towards further substitution, the product distribution on rearrangement should be greatly simplified. Thus, heating 3-mesityl-1,2,3triphenylcyclopropene (Ic) at $190-200^{\circ}$ for $2 \mathrm{hr}$. afforded two cleanly separable indene products, m.p. $179-181^{\circ}$ and $131-132^{\circ}$, in about equal amounts. The higher-melting product $\left[\lambda_{\max }\right.$ $\left(\mathrm{CHCl}_{3}\right) 330(\mathrm{sh}), 315(\mathrm{sh}), 307$, and $240 \mathrm{~m} \mu$; n.m.r., complex multiplet at $\tau 2 \cdot 84(17 \mathrm{H})$, sharp singlet at $4 \cdot 73(1 \mathrm{H})$, doublet centred at $7 \cdot 65(6 \mathrm{H})$, and sharp singlet at $8.12(3 \mathrm{H})]$ has been identified as 3 mesityl-1,2-diphenylindene (IIC). The nonequivalence of the ortho-methyl groups of the pendant mesityl ring in (IIc) is attributed to restricted rotation of this group. The lower-melting isomer $\left[\lambda_{\max }\left(\mathrm{CHCl}_{3}\right) 297.5\right.$ and $240 \mathrm{~m} \mu ;$ n.m.r., complex multiplet at $\tau 2 \cdot 80(17 \mathrm{H})$ with sharp singlets at $7 \cdot 74$ $(3 \mathrm{H}), 8 \cdot 10(3 \mathrm{H})$, and $8 \cdot 30(3 \mathrm{H})]$, at first suspected to be the 1-mesitylindene (IVc), is now tentatively assigned the 1,4,6-trimethyl-1,2,3-triphenylindene structure $(V)$ on the basis of the highly nonequivalent nature of the methyl groups in the n.m.r. spectrum, the stability to base under aerobic conditions, and the mode of formation involving a 1,5-methyl shift from isoindene intermediate (VI).<smiles>Cc1ccc2c(c1)C(C)(c1ccccc1)C=C2</smiles>

(V)<smiles>Cc1ccccc1C12C=CC=CC1=CC=C2</smiles>

(VI)
The formation of $(\mathrm{V})$ on thermal rearrangement of (Ic) is taken as evidence for the diradical nature of the rearrangement, despite the fact that we have not, as yet, been able to trap the intermediate by conventional means. Thermal rearrangement of (Ia) in cumene gave almost quantitative yields of (IIa) with no evidence of bicumyl formation. Similar trapping attempts with methyl acetylenedicarboxylate were unsuccessful.

The above arylcyclopropene-indene rearrangements are analogous to the better studied vinylcyclopropane rearrangements, ${ }^{1}$ but to our knowledge there is no report in the literature of corresponding indane formation from arylcyclopropanes. For the purpose of comparison, cis-1,1,2,3-tetraphenylcyclopropane (VII) was synthesized in $78 \%$ yield by di-imide reduction ${ }^{4}, 7$ of (Ta). Heating (VII) in diphenyl ether at $200^{\circ}$ for $3 \mathrm{hr}$. resulted in quantitative rearrangement to the trans-isomer (VII) ${ }^{8} \quad$ No olefin or indane products were detected even at higher temperatures. The first-order rate parameters for the isomerization (Table) were determined by the n.m.r. technique, integrating the respective singlets for the cis- and transcyclopropyl protons.

From the Table it is seen that the isomerization

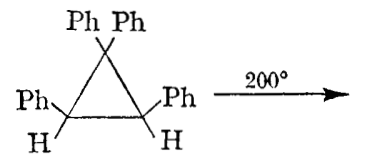

(VII)

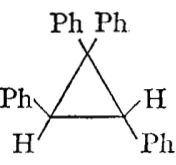

(VIII)

$\ddagger$ Satisfactory analytical data were obtained for all new compounds reported. 
TABLE

\section{Kinetic parameters in diphenyl ether}

\begin{tabular}{|c|c|c|c|c|}
\hline Isomerization & $T\left({ }^{\circ} \mathrm{C}\right)^{\mathrm{b}}$ & $10^{5} k\left(\mathrm{sec} .^{-1}\right)$ & $E_{\mathrm{a}}(\mathrm{kcal} / \mathrm{mole})$ & $\log A$ \\
\hline$(\mathrm{I}) \rightarrow(\mathrm{II})^{\mathrm{a}}$ & $176 \cdot 7$ & $0.450 \pm 0.035$ & $40 \pm 1$ & $14 \cdot 0 \pm 0.5$ \\
\hline$(\mathrm{VII} \rightarrow \quad(\mathrm{VIII})$ & $\begin{array}{l}205 \cdot 1 \\
176 \cdot 6 \\
155 \cdot 5\end{array}$ & $\begin{array}{l}5 \cdot 76 \pm 0.28 \\
4.93 \pm 0.08 \\
0.602 \pm 0.009\end{array}$ & $38.4 \pm 0.2$ & $14 \cdot 3 \pm 0 \cdot 1$ \\
\hline
\end{tabular}

a solvent contains $12 \%$ (by wt.) of quinoline; ${ }^{\mathrm{b}} \pm 0 \cdot \mathbf{2}^{\circ}$.

of (VII) proceeds at a rate approximately ten times that for the rearrangement of (Ia) to (IIa). While this rate difference is small, it is significant that opening of the cyclopropane ring is occurring faster than that for the more highly strained cyclopropene. This result must have some bearing on the nature and stability of the intermediates involved, and argues against a concerted process. In terms of the radical mechanism, however, homolytic cleavage of (VII) would lead to a more stable diradical than that obtained from (Ia), accounting for the observed kinetic data and lack of indane formation from the arylcyclopropane.

We are grateful to the National Science Foundation for partial support of this work, and to Mr. James Todd, who carried out some of the initial experiments on the thermal rearrangement of tetraphenylcyclopropene.

(Received. July 13th, 1967; Com. 728.)

1 R. Breslow in "Molecular Rearrangements", Part I, ed. P. de Mayo, Interscience, New York, 1963, pp. 236-261.

${ }^{2}$ R. Breslow and P. Dowd, J. Amer. Chem. Soc., 1963, 85, 2729; R. Breslow, P. Gal, H. W. Chang, and L. J. Altman, ibid., 1965, 87, 5139.

${ }^{3}$ M. A. Battiste and R. B. Grubbs, in preparation.

4 M. A. Battiste, Tetrahedron Letters, 1964, 3795.

5 E. P. Kohler, Amer. Chem. J., 1908, 40, 217.

${ }^{6}$ W. R. Roth, Tetrahedron Letters, 1964, 1009.

${ }^{7}$ R. S. Dewey and E. E. van Tamelen, J. Amer. Chem. Soc., 1961, 83, 3729; E. W. Garbisch, jun., S. M. Schildcrout, D. B. Patterson, and C. M. Sprecher, ibid., 1965, 87, 2932.

${ }^{8}$ An independent synthesis of (VIII) by photocyclization of the 1,3,3,3-and 1,1,3,3-tetraphenylpropenes has been previously reported by G. W. Griffin, A. F. Marcantonio, H. Kristinsson, R. C. Petterson, and C. S. Irving, Tetrahedron Letters, 1965,2951 . These authors also observed the thermal isomerization of (VII) to (VIII), and we are indebted to them for a complete interchange of compounds and structural data. 